Flavio Corradini, Francesco De Angelis*, Andrea Polini, Carlo Castagnari, Jacopo de Berardinis and Giorgio Forcina

\title{
Tangramob: An Agent-Based Simulation Framework for Validating Urban Smart Mobility Solutions
}

https://doi.org/10.1515/jisys-2018-0321

Received August 3, 2018; previously published online January 26, 2019.

\begin{abstract}
Estimating the effects of introducing a range of smart mobility solutions within an urban area is a crucial concern in urban planning. The lack of a simulator for the assessment of mobility initiatives forces local public authorities and mobility service providers to base their decisions on guidelines derived from common heuristics and best practices. These approaches can help planners in shaping mobility solutions; however, given the high number of variables to consider, the effects are not guaranteed. Therefore, a solution conceived respecting the available guidelines can result in a failure in a different context. In particular, difficult aspects to consider are the interactions between different mobility services available in a given urban area and the acceptance of a given mobility initiative by the inhabitants of the area. In order to fill this gap, we introduce Tangramob, an agent-based simulation framework capable of assessing the impacts of a smart mobility initiative within an urban area of interest. Tangramob simulates how urban traffic is expected to evolve as citizens start experiencing newly offered traveling solutions. This allows decision makers to evaluate the efficacy of their initiatives, taking into account the current urban system. In this paper, we provide an overview of the simulation framework along with its design. To show the potential of Tangramob, three mobility initiatives are simulated and compared in the same scenario. This demonstrates how it is possible to perform comparative experiments so as to align mobility initiatives to the user goals.
\end{abstract}

Keywords: Smart mobility, agent-based traffic simulations, smart urban planning.

Classification: 68T42 Agent technology

\section{Introduction}

According to the United Nations [17], in 2016, the world's population was 7.4 billion inhabitants, and about $54.5 \%$ of them lived in urban areas. Despite all the benefits historically brought by urbanization, like poverty reduction, longer life expectancy, and economic wealth, such an uncontrolled demographic growth is pushing cities to deal with several management problems. Focusing on urban mobility, transport infrastructures are close to saturation, and this comes with a bunch of problems like car dependence, spatial footprint, traffic congestion, and air and noise pollution. Novel smart mobility solutions need to be introduced, and investments have to be carefully assessed in relation to their effective potential to improve the mobility ecosystem. These initiatives are generally shaped, and their adoption assessed, considering common guidelines and best practices. Nevertheless, it is not seldom the case that the observed effects, after the concrete deployment of a solution, are not satisfactory. In particular, there are two complex aspects that are difficult to assess when

\footnotetext{
*Corresponding author: Francesco De Angelis, School of Science and Technology, University of Camerino, via Madonna delle Carceri 9, Camerino MC, Italy, e-mail: francesco.deangelis@unicam.it. https://orcid.org/0000-0001-8827-6033 Flavio Corradini, Andrea Polini and Carlo Castagnari: School of Science and Technology, University of Camerino, via Madonna delle Carceri 9, Camerino MC, Italy

Jacopo de Berardinis: School of Computer Science, University of Manchester, Oxford Road, Manchester, M13 9PL, UK Giorgio Forcina: School of Innovation Design and Technology, Mälardalen University, Västerås, Sweden
} 
following such approaches to planning. The first one relates to how the new mobility solution will interact with the already available ones, whereas the second one relates to acceptance by citizens. Indeed, as shown by many reports [15], there are many cases in which the adoption of a smart mobility initiative (SMI) did not bring the expected benefits.

These considerations motivated us to develop a novel simulator named Tangramob. Tangramob is an agent-based simulation framework capable of assessing the impacts of an SMI (i.e. a range of either homogeneous or heterogeneous smart mobility services) within an urban area of interest. We can envision this framework as a tool aimed at urban planners and transport companies to geographically placing a number of smart mobility services (e.g. carsharing, bikesharing, and so forth) within a city and configuring each of them according to their expectations and budget. This will complement already available approaches in the definition and shaping of the smart mobility solution to adopt.

The contribution of our work is an easy-to-use simulation framework to help decision makers in planning SMIs. Tangramob, built over an agent-based formalization of urban mobility, is able to assess the effectiveness and human acceptance of SMIs. This work is distinguishable from other approaches in the following aspects: (i) support of intermodal and multimodal transport services, (ii) characterization of human behavior for traveling choices, (iii) adaptability and support of different geographic areas, and (iv) possibility to reflect the diversities of commuters with respect to their personal characteristics (e.g. gender, age, travel demand). Tangramob is an open-source project, and we expect an active contribution and interest from the smart mobility community. It is built over MATSim, a powerful traffic simulator [11]. Tangramob is aimed at all people involved in defining and planning new mobility services: urban planners, who are in charge of improving urban mobility; transport companies, which need to ponder their investments; and researchers, who aim at testing and validating new solutions.

The rest of the paper is organized as follows: Section 3 outlines the idea behind the Tangramob simulator and how it is expected to address the research problem. Section 4 provides an overview of the agent-based model (ABM) of Tangramob, and Section 5 describes its architecture. Section 6 proves the effectiveness and potentialities of Tangramob by reporting an example of its use. Section 2 shows the current attempts in supporting urban planners and mobility service providers. Finally, Section 7 reports some conclusions and opportunities for future work.

\section{Related Work}

To the best of our knowledge, the state of the art does not provide any easy-to-use tool for assessing the impacts of SMIs, in particular when several mobility services are considered. Several tools exist as traffic simulators under specific constraints and single service simulation.

MATSim [11] is an activity-based multiagent simulation framework for implementing large-scale agentbased transport scenarios. It has a structure based on a queue-server model to reproduce traffic using a mesoscopic model. Although car-following approaches are not used, the authors state that "the model should be somewhat realistic, so that comparisons to real-world traffic are possible" [4]. In effect, the creators of MATSim rigorously demonstrated in Ref. [11] how simulated traffic resembles the actual one. MATSim is usually used to simulate a single smart mobility solution. Therefore, there is still no way of assessing the impact expected after the introduction of a heterogeneous range of smart mobility solutions. Other than MATSim, another noticeable software tool for traffic simulations is SUMO [2]. SUMO is an open-source traffic simulation tool that includes demand modeling. SUMO is used for vehicular communication, route choice and dynamic navigation, traffic light algorithms, evaluation of surveillance systems, emissions and noise modeling, and person-based intermodal traffic simulation. Unlike MATSim, its simulation happens at the microscopic level and without using agent-based technology. Using SUMO, Jabbarpour et al. [12] aimed at reducing fuel consumption and consequently $\mathrm{CO}_{2}$ emissions. They were able to find the least congested shortest paths in order to reduce vehicle traffic congestion and pollutant emissions, evaluating the results according to various criteria like average travel time, speed, distance, and vehicle density, along with road map segmentation that focuses on reducing fuel consumption as much as possible. Making use of SUMO, the 
hybrid vehicle routing model is evaluated and validated for the city of Kuala Lumpur (Malaysia) through a series of traffic simulation experiments. Other than MATSim and SUMO, in this simulator landscape, we look with interest, for future functionality and result comparison, at the simulators discussed below.

SimMobility [1] integrates behavioral models to predict the impact of multimodal mobility demands on transportation networks and services. It simulates how people will react in the uncertain future using an activity-based approach. The simulation is characterized by a multiscale approach: a short-term approach looks at microscopic simulations, a mid-term approach looks at transportation demands using an aggregate model to move people and vehicles, and a long-term approach is used to capture land use and long-term effects.

VISSIM [6] simulates traffic including driver behavior with a following model, lane changing, and nonlane-based behavior, thus providing a more complex traffic model with respect to MATSim and suitable for other types of simulations like traffic patterns, rail- and road-related public transport, pedestrians, and cyclists.

SMART (Scalable Microscopic Adaptive Road Traffic Simulator) [16] overcomes the problem of slow simulation, performing a distributed microscopic traffic simulation on multiple independent processes running parallel. It provides a car-following model and a lane-changing model, and supports multiple vehicle types including bus and tram.

Other simulators are specialized for a specific task or context; for example, ABSTUR [8] simulates how many tourists sign up for a tourist route considering the features of some routes in a city and of the tourists. The simulator receives input from a set of routes and a certain number of tourists of different types, and provides the number of tourist people signed up for each route after the simulation. This allows experts to avoid collections of routes that are overcrowded or non-profitable, and allows building a recommender system for final users. ABSCEV [9] aims to simulate queues of fast-charging stations for electric cars to reduce waiting times. Using social coordination mechanisms, the agent-based simulation framework simulates the effects of different coordination policies in the route planning of electric drivers for charging their vehicles on their trips. Although different in the final aim, these works show the use of agent-based systems as a decision support for smart mobility in a city environment.

\section{Tangramob Simulator}

Tangramob is an agent-based simulation framework that intends to support public and private decision makers in the task of shaping SMIs for a specific urban area of interest. It can be considered as a decision support system for smart mobility validation, focusing on the ability to capture and reproduce the mobility behavior of each single commuter belonging to the selected sample population. For this purpose, Tangramob is organized as a simulation environment that the urban planner can easily use in order to understand if introducing an SMI, i.e. a collection of mobility services, can improve the traveling experience of citizens as well as the performance of the urban transport system. As the simulator is based on an ABM, for each person in the sample population, represented as an autonomous reasoning agent, we can observe whether or not it will make use of the new mobility services. These fine-grained results also provide users with a measure concerning the expected adoption rate of the simulated mobility initiative, so as to figure out beforehand if the initiative can potentially succeed or not. Technically, a Tangramob simulation requires four inputs:

- The urban road network of the area under study.

- A representative population of the area with the mobility agendas of people. An agenda summarizes what a person does during an ordinary working day (i.e. activities) and how he/she moves from one place to the next one (i.e. legs).

- The description of the mobility services already offered by the city (public transport timetable, etc.).

- The SMI to evaluate, i.e. a list of geographically located containers (called tangrhubs) of one or more smart mobility services. Each smart mobility service belongs to a tangrhub, and it comes with a number of mobility resources (e.g. vehicles) as well as a service charge (i.e. cost per kilometer and cost per hour). 
It is worth mentioning that the definition of an agent population is certainly the most complex and critical information to supply, in particular in relation to profiles and details on daily travels. The more the population is representative of the reality of interest, the more the results of the simulation can be considered a good approximation. Strategies for the derivation of a population are out of scope for this paper. Nevertheless, different sources are available to define a representative synthetic population. Relevant data can be certainly collected from periodic census or questionnaires distributed to a sample population. Particularly effective nowadays is mobile crowdsensing [10], which uses mobile apps developed for large-scale sensing and involves the contribution from a crowd of people that behave as probes of the dynamics of the mobility in the city [13].

Starting from the provided information, the execution of Tangramob can be thought of as performing a comparative experiment. The experiment consists in introducing the SMI (i.e. applying the treatment) into the urban area of interest (i.e. the treated system) while observing the same reality as it is today, namely with no SMI (i.e. control system). In the end, we can observe how these systems differ with respect to the following measures:

- Travel distance, in meters, referring to the distance traveled by a commuter;

- Travel time, in seconds, referring to the time spent traveling for a commuter;

- $\mathrm{CO}_{2}$ emissions, in grams, referring to the quantity of $\mathrm{CO}_{2}$ produced by each commuter according to the used means of transport;

- Cost of mobility, in euros, referring to the cost of mobility for a commuter;

- Urban traffic levels, referring to the number of traveling vehicles on each road at a given moment in a time slot. This statistic represents the road infrastructure with respect to the traffic congestion levels in a time slot.

Such a comparison would allow the user to understand if the proposed mobility initiative is in line with their expectations. In case they are not satisfied with the achieved results, the user can change the configuration of the mobility initiative (e.g. relocating tangrhubs, adding/removing tangrhubs, modifying the parameters of a mobility service, and so forth) in order to repeat the experiment as before.

\subsection{Tangrhubs}

In Tangramob, the actual placement of smart mobility services within the urban area under study is made possible by tangrhubs. A tangrhub can be defined as a geo-located entity providing citizens with one or more mobility services. A tangrhub collects one or more smart mobility services, each of which is offered by either private or public providers. For instance, a carsharing service provided by two different companies results in two different characterizations of resources and their usage deployed within the tangrhubs of interest. Considering the typical urban conformation, such a flexible and modular abstraction allows urban planners to represent all existing transport facilities like railway stations, bus stops, and so forth, and to introduce intermodality among the mobility services. Indeed, a bus stop could be represented as a tangrhub where only the bus service is available.

Examples of smart mobility services that the user can add to a tangrhub are dynamic public transport, shared transport services (e.g. carsharing, bikesharing), dynamic ridesharing, autonomous taxis, and so forth [5]. However, each smart mobility service provided by a tangrhub must belong to only one of the following service types: intrahub services, used for moving people inside the relevance area of the hub, and interhub services, for moving commuters from between tangrhubs that support the same mobility service type.

From the simulator's perspective, we can think of a tangrhub as an entity with which people interact as soon they need to travel. As a result of such interactions, tangrhubs are expected to collaborate with each other in order to provide commuters with a list of traveling solutions. Thus, it is up to commuters to evaluate and choose the most suitable solution according to their needs and preferences. 


\subsection{SMI}

According to the concept of tangrhub seen before, shaping an SMI is about placing a number of tangrhubs within the urban area of interest, adding one or more smart mobility services to each of them, and providing a specific characterization for the added mobility services. Thus, it is up to the user (e.g. an urban planner) to design a list of candidate SMIs according to the goals and the available resources of his/her local authority.

To define a smart mobility service for a tangrhub, such as carsharing, the user has to specify the service type (i.e. intrahub or interhub), the initial number of vehicles, and the service charge (i.e. cost per kilometer, per hour, and fixed) and other parameters depending on the type of vehicles. Therefore, in Tangramob, a mobility service provided by an organization is represented as a whole - as the sum of all services made available by the same organization within the selected tangrhubs. It is worth noticing that the cost of a mobility service does not need to correspond to a real currency. In fact, we can consider cost in terms of "points," as such an approach fits the idea of mobility as a service [3, 7].

These cost-related parameters are expected to affect the mobility decisions of commuters. More precisely, commuters are more inclined to choose the most convenient services, i.e. the ones with the greatest efficiency/cost trade-off.

\subsection{Tangramob Commuting Patterns}

As new mobility opportunities are introduced, commuters are expected to change their daily commuting patterns. A commuting pattern is the intermodal representation of how a person moves from one place to another. Such a trip can be either simple (e.g. by car) or more complex (e.g. by a combination of travel modes). An example of commuting pattern is a route provided by Google Maps. In Tangramob, the complexity of these patterns can be limited due to the direct interconnection of tangrhubs via their interhub mobility services. Commuters are never offered with traveling solutions made up of more than three sub-trips (Figure 1A).

Analogously, the second and the third classes (Figure 1B,C) represent a combination of two modal trips performed either by intrahub services or by personal traveling modes. Finally, the last class (Figure 1D) corresponds either to a direct trip (e.g. by car, walk) or to the case a single interhub service is used.

It is worth saying that commuting patterns are not made up of more than three sub-trips, as we assume that each tangrhub is directly linked with all the other ones by means of at least one mobility service. The schema of such a kind of configuration looks like a complete graph in which all the nodes have a direct link to all the other nodes. Although this assumption could seem too strong, we aim at reducing the number of different means of transportation used by the commuters for their daily journeys, and, as a consequence, increase their travel comfort.

A

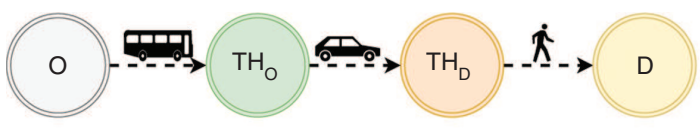

C

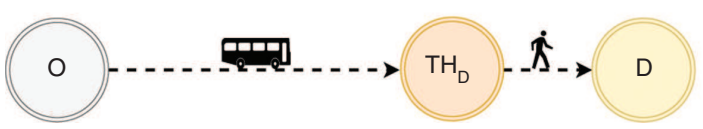

B

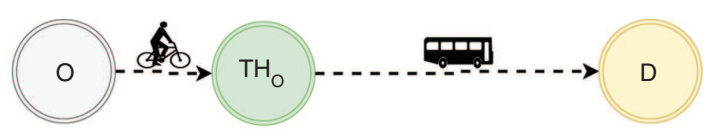

D

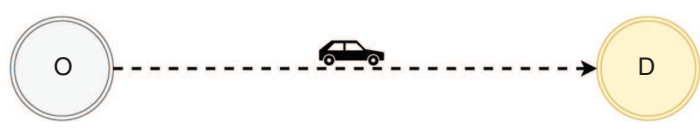

Figure 1: Examples of Commuting Patterns in Tangramob.

(A) Three-trip path, (B) two-trip path I, (C) two-trip path II, and (D) direct path. 


\section{Tangramob ABM Overview}

Starting from the idea of Tangramob, we present the ABM on which it is conceived. The Tangramob ABM is composed of two agent types: commuter and tangrhub. A commuter agent is the computational representation of a single person that is part of the sample population under study. Every commuter agent comes with some relevant personal characteristics, like gender and age, affecting the outcomes of the actions taken during the simulation. These effects also impact on the behavior of commuters. For instance, an elderly person will be less prone to travel by bicycle for long trips, as this would take too long for him/her. More important, every commuter has a personal mobility agenda, i.e. a sequence of daily activities (e.g. home, work, etc.) interleaved by mobility segments that tell how the agent manages to get from one activity location to the next one.

A tangrhub agent can be defined as a local mobility service provider with the ability to improve its services as the simulation iterates; in the real world, this active behavior might correspond to a daily enhancement.

Both agents live and operate, albeit with different perceptions, in a composite environment that is made of three different spaces: the temporal space, the geographical space, and the smart mobility services' state space. Specifically, the temporal space reflects the passage of time in seconds. The geographical space can be defined as the directed weighted graph resulting from the road network infrastructure of the urban area under study; in particular, nodes represent intersections and edges denote streets. Such a space is the actual core of the transport simulation, as the physical limitations of the road infrastructure can create bottlenecks and delays as people move with a certain pace. Finally, the last sub-environment is meant to represent the status of all the smart mobility services that are currently provided by tangrhubs. This space can be conceived as a tuple space, where the status of each mobility service is broken down into a number of smaller sub-states. For instance, the status of a carsharing service can be expressed as the combination of the states of all its vehicles.

This complex environment allows agents to perform actions that can eventually alter the state of affairs of one or more sub-environments. In particular, every time a commuter needs to move from one place to another, an interaction with the surrounding tangrhubs takes place. During this interaction, a smart mobility negotiation occurs: the tangrhubs collaborate with each other in order to provide the commuter with a number of traveling alternatives. A traveling alternative can be thought of as a combination of one or more (up to three) mobility segments, each of which can involve a smart mobility service and is based on the Tangramob commuting patterns discussed in Section 3.3. Next, the commuter agent will perform a decision-making process so as to select the traveling alternative that is expected to maximize his/her performance criteria.

The alternative selection process is organized as follows: first, every single traveling alternative is evaluated according to the expected performance of each segment it is made of; then, the cost is introduced to influence such preference-ordered rank; finally, a traveling alternative is selected and then simulated. Once the commuter agent has reached his/her final destination, he/she is expected to assign a score to every single commuted mobility segment to record its traveling experience so as to make more informed decisions for the next iterations.

As soon as a traveling alternative is chosen, the involved tangrhubs will reserve the required mobility services so that the commuter can start his/her journey. Finally, once the commuter has reached his/her destination, he/she will be asked to leave a feedback for each smart mobility service used. The behavior of a commuter revolves around four actions: (i) synchronizing his/her mobility agenda with the closest tangrhubs, to obtain a list of traveling alternatives for reaching the location of the forthcoming activity; (ii) choosing a traveling alternative out of the proposed ones; (iii) performing the chosen traveling alternative; and finally (iv) leaving as many feedback as the number of mobility services used in the course of the day. A commuter will then try to maximize his/her traveling experience by minimizing the travel time, covered distance, emissions, and cost of mobility. More precisely, this is done by selecting the traveling alternative that is expected to optimize such criteria from time to time. The tangrhub agent has the following two goals: to maximize the traveling experience of commuters and minimize the number of mobility resources for each service. Thus, in order to achieve these objectives, a tangrhub can perform the following actions: (i) build a list of traveling alternatives 
in collaboration with other tangrhubs; (ii) provide a commuter with a list of valid traveling alternatives; (iii) update the status of its own mobility services; and (iv) improve and optimize its own mobility services.

The tangrhub's service adaptation process is made possible by commuters' feedback. In particular, each feedback qualifies the traveling experience of a commuter using a specific mobility service. Collecting and averaging all the feedbacks of a mobility service can give a metric concerning the performance of that service, thereby contributing to its improvement and optimization. For instance, if all the daily collected carsharing feedbacks are negative, a tangrhu $b$ would have a valid indicator of such an inefficiency to run for cover. Therefore, the purpose of a feedback is twofold: on the one hand, it pushes the commuter agent to reason about the quality of the services to make more informed decisions for the next iterations; on the other hand, it enables tangrhubs to align to the needs of the population.

Tangramob simulations are thus iterative; each iteration corresponds to a typical day in which commuters experiment the introduced smart mobility services and record their performance, while tangrhubs can improve their services iteration by iteration. This time-evolving behavior, driven by feedback, enables commuters to make more informed decisions every time they are offered a list of traveling alternatives. Therefore, commuters are modeled as proactive agents, as there is need for an iteration-persistent memory structure, i.e. a knowledge base, to implement such an experience-based learning capability. With that idea, the decision-making process of commuters exploits their personal knowledge base in order to evaluate the expected score of a traveling alternative, thanks to the experience accumulated from past iterations. This is achieved by updating the knowledge base, by means of a Hebbian-like learning function. This will permit to gradually accumulate scores so as to let the commuter maturate an experience-based perception for every segment. Similarly, tangrhubs are modeled as self-adaptive agents that can use different strategies and optimization methodologies to enable their travel-improving behavior at the end of each iteration and by means of feedbacks.

\section{Design and Implementation Overview}

Considering the agent-based nature of Tangramob, the framework has been developed on an already validated and robust agent-based traffic simulator named MATSim [11]. Such a design choice is due to the fact that it is possible to represent the characterizations and behaviors of both our agent types in MATSim. Moreover, such a simulator can be adapted to support all the sub-environments of the model, allowing Tangramob to evaluate the performance criteria as outcomes from the interactions among such spaces and agents.

\subsection{Multiagent Transport Simulation: MATSim}

MATSim [11] is an activity-based multiagent simulation framework for implementing large-scale agent-based transport scenarios. It is an open-source project implemented in Java under the GNU's Not Unix public license. As in Figure 2, the framework consists of several modules that can be combined, used standalone, or replaced by own implementations. MATSim is designed to model a single day, and it is based on a co-evolutionary approach in order to reproduce real-life scenarios. Every agent repeatedly optimizes its daily activity schedule while in competition for space-time slots with all other agents on the transportation infrastructure. This optimization follows an iterative process, and it is based on different choice dimensions such as route selection, time choice, and mode choice. A MATSim run consists of a number of iterations repeated in a cyclical manner.

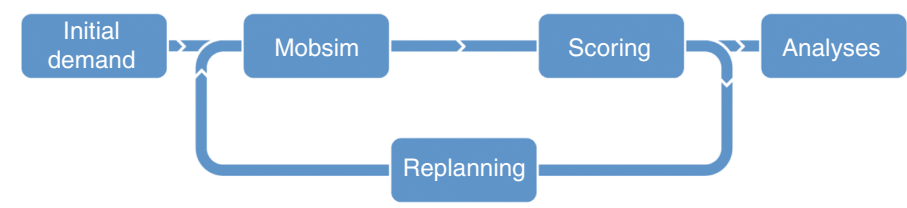

Figure 2: MATSim Modules. 
MATSim can be applied in large scenarios. We show an example considering a small city in the paper. Nevertheless, scalability to bigger cities should not be much of a problem as MATSim simulations of large-scale agent-based micro-simulation models have been proven scalable [18]. An experiment made by MATSim developers with 1.62 million agents and 163,000 links in the area of Zurich City were simulated in about 20 min in a machine with 128 GB RAM and 8 dual-core AMD Opteron central processing units (CPUs). Also, the Switzerland traffic was modeled in about $3 \mathrm{~h}$ for a single MATSim iteration: 1 million roads and 7.3 million agents clearly showed that large-scale, multiagent micro-simulation can be reasonably used.

\subsection{Tangramob Meets MATSim}

Our framework has been implemented on top of MATSim, taking advantage of its flexible and modular architecture and trying to maintain the same design principles. We redefined and extended the behavior of some original MATSim modules as in Figure 3, whereas other remarkable contributions were introduced in such a way to capture all the features of the Tangramob ABM of Section 4.

In particular, the module initial demand, in which the simulation input data are collected and validated, is integrated with the specification of the SMI, describing the locations of the tangrhubs on the map as well as the list of the mobility services available on each of them. Making this integration possible required us to implement the concept of the tangrhub agent, a new static but active entity that is responsible both for managing and offering new traveling opportunities to the nearby population, and for managing the associated mobility services, which can be seen as services provided by private or public companies/organizations and that overall constitute the infrastructure of the SMIs available in the urban area.

The mobsim module, specialized in simulating the urban traffic, has been integrated with the MATSim "multimodal" extension, which allows dealing with different transport modes as well as simulating the overtaking of vehicles. This way, Tangramob can also evaluate the impact on the urban system caused by unconventional kinds of vehicles (e.g. scooters, bicycles, etc.). For this purpose, we redesigned the original

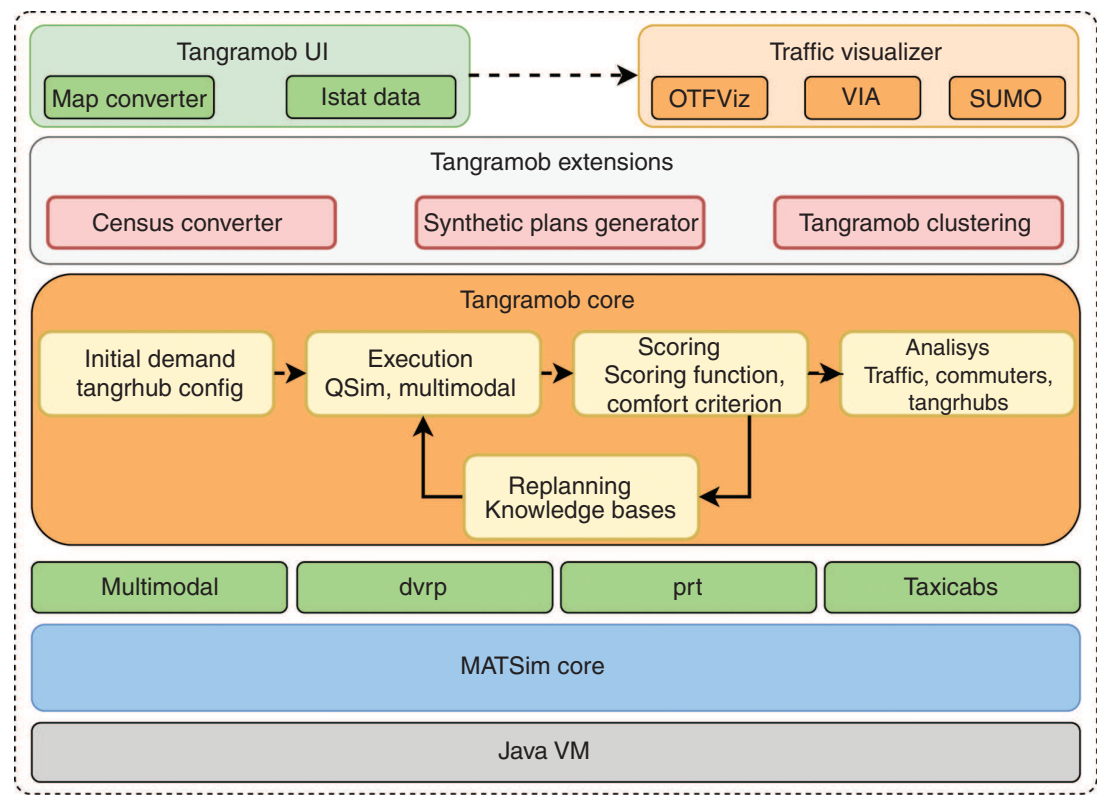

Figure 3: Tangramob Architectural Overview.

Colors are used to distinguish either the scope or the source of each module: green boxes refer to software contributions that we use both from the MATSim project [11] (multimodal, dvrp, etc.) and from the codebase of Tangramob (map converter, istat data); orange boxes denote core software components that we extended and re-implemented (i.e. yellow modules within the core block) or used as they are (i.e. OTFViz, Via, SUMO); red boxes refer to single extensions to the core of Tangramob, each of which provides extra features for facilitating the configuration of a simulation. Continuous lines describe the execution flow of a simulation, whereas dashed lines denote software dependencies. 
concept of MATSim's vehicles, and we introduced the characterization of mobility services with the ability to manage such vehicles. Furthermore, our characterization takes into account the most relevant vehicle features, like dimension, velocity, fuel type, and consumption; all these specifications are expected to impact on the traffic simulation, especially for what concerns travel delays and times, and thus are relevant information in relation to the mobility decisions of commuters.

Concerning the scoring module, Tangramob still exploits the original Charypar-Nagel scoring function [11]. This allowed us to validate the new learning process of Tangramob, exploiting the existing MATSim validation work.

The replanning phase designed for Tangramob is completely different from that followed by MATSim. Whereas MATSim adopts a co-evolutionary algorithm, our framework is based on a reinforcement learning approach, allowing each commuter to evaluate his/her past traveling experience in order to improve his/her daily personal mobility. This is made possible by the implementation of iteration-persistent memory structures, which every commuter can exploit as a knowledge base, in order to accumulate the score given for each mobility service used during the simulation. Thus, the score of a service acts as a reward for the action of choosing that service for a certain trip. Such a different approach allows commuters to maximize the expected utility of their mobility decisions. In particular, during the last iteration of the simulation, each commuter will decide to either use the new mobility services or not to accept the mobility initiative, according to the collected knowledge.

Finally, the analysis module has been integrated with new statistical collectors to gather all data useful to compare the legacy urban mobility with the one after the introduction of a SMI. Some statistics correspond to the agents' performance criteria described in Section 4, and others are focused on the urban system as a whole. We aimed at collecting the following statistical data: (i) urban traffic levels, (ii) $\mathrm{CO}_{2}$ emissions, (iii) traveled distances, (iv) travel times, (v) land use levels, (vi) cost of mobility, (vii) number of adopters, and (viii) resource usage level.

The resulting architecture is fully extensible in every layer, providing the possibility to develop extensions over both the MATSim layer and the Tangramob layer.

\section{Tangramob: An Example of Use}

In order to show an example of use of Tangramob, we report some experiments performed on a real scenario in the city of Ascoli Piceno (Italy). Ascoli Piceno is a small city with about 50,000 inhabitants over $158 \mathrm{~km}^{2}$ and several other thousands of people who live in near places outside the city perimeter. Considering the novelty of Tangramob, a comparison of our results with those of other simulators in the literature would not be consistent with the objectives of this experiment. More precisely, as better detailed in Section 2, the existent simulators are mainly focused on tracing the expected evolution of traffic in response to changes in the road network infrastructure. Even though a few of them can simulate mobility services and their potential impact on the urban system, to the best of our knowledge, none of them considers the use of multiple mobility services as commuting alternatives. Moreover, conversely to other contributions, Tangramob does not assume any fixed traveling behavior from commuters, as the choice of a combination of services to use depends on the experience accumulated during iterations.

As depicted in Figure 4, the network represents all the city roads and infrastructures including the city center and the roads that connect the city with other places. From the statistical data on the urban population available from the website of the municipality, we identified 15 areas that can be served by 11 tangrhubs. Then, for each area, we created a sample population that is intended to emulate the traveling needs of the corresponding inhabitants according to their jobs. The mobility agendas of the synthetic population considered in this study were thus obtained directly from the inferred home and work locations of the inhabitants living in each area.

In the experiment, we modeled the whole population considering the suburbs with 56,000 agents. Using the statistics of the municipality, we built a normally distributed population age with $45 \%$ in the range of 
25-49 years (52\% female and 48\% male). These parameters are expected to affect the act of traveling of commuters, thus impacting on their score. Basically, mobility agendas were organized with three daily activities in the following order: home, work, home. Thus, a commuter moves from home to a workplace in another area and vice versa. For the sake of clarity, we considered in this experiment as work each kind of activity different to stay at home. We also did not consider multitrip commutes.

As a typical real-case scenario, peak activity hours can be split into two different moments: 8 a.m. commuters start moving toward the workplaces, while at 16:00 commuters come back home from work. The first activity in the morning is distributed in the 5:00-13:00 time slot with 45\% included in the 07:00-08:00 h. The homecoming happens at the end of the work activity. That time is modeled using a Gaussian distribution centered over a 6-h duration.

In this scenario, we aimed at investigating the impacts of three different SMIs that integrate transport services: a bikesharing, a carsharing, and a scootersharing service. All vehicles used were zero emissions. Although Tangramob could support the integration of public transport, we intentionally decided to restrict the focus of our study to those commuters who do not make use of sustainable mobility solutions, such as those traveling alone by personal car. We used 11 tangrhubs in the city areas, as several locations in the city center can be served by the same hub. Each hub is characterized as in Table 1. For readability, we used the same resources for each tangrhub in this example.

As shown in Table 1, each SMI shares the same number of tangrhubs, each of which is provided with the same choice set of mobility services. Even the geographical location of tangrhubs is the same for all the initiatives, and it is denoted by the triangles depicted in Figure 4. For each tangrhub, we specify the dimension of the fleet the hub manages at the start of the simulation, and its total capacity to store vehicles is set at $25 \%$ more than the initial fleet. What differs among these initiatives is just the number of mobility resources, which, in this case, correspond to the vehicle fleet of each service.

For each mobility service, we specified the costs. For this experiment, we set the costs of the chosen mobility services, which in turn were set according to the actual average service charges in Europe, as summarized in Table 2.

As argued in Section 3, understanding how the proposed SMIs impact both commuters and the transport system requires a comparative experiment. In particular, considering that commuters in this scenario are used to moving by private cars, we first simulated the current urban mobility (i.e. the pre-SMI simulation),

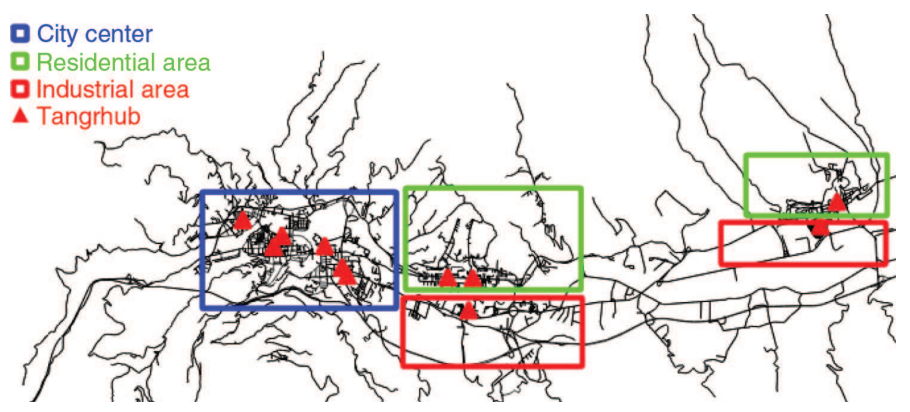

Figure 4: Ascoli Piceno Network with Tangrhub Positioning for SMIs.

Table 1: Grid Network: Tangrhub Experimental Setup.

\begin{tabular}{llrrr}
\hline & & & Fleet \\
\cline { 2 - 5 } & Mobility services & SMI-1 & SMI-2 & SMI-3 \\
\hline TH & Bikesharing & 10 & 10 & 50 \\
& Carsharing & - & 10 & 50 \\
\multirow{4}{*}{ Total } & E-scootersharing & - & 10 & 50 \\
& Bikesharing & 110 & 110 & 550 \\
& Carsharing & - & 110 & 550 \\
& E-scootersharing & - & 110 & 550 \\
\hline
\end{tabular}

TH, TangrHub. 
Table 2: Grid Network: Mobility Services’ Costs.

\begin{tabular}{lrrr}
\hline & Cost per hour & Cost per kilometer & Fixed cost \\
\hline Bikesharing & 0.5 & 0 & 0.01 \\
Carsharing & 13 & 0.1 & 0.01 \\
E-scootersharing & 2.5 & 0.1 & 0.01 \\
\hline
\end{tabular}

then we simulated each SMI separately (i.e. SMI-1, SMI-2, and SMI-3 simulations). Thereafter, we compared these simulations with respect to the following variables: traveled distance, travel time, $\mathrm{CO}_{2}$ emissions, cost of mobility, and urban traffic levels; all these values were collected during the last iteration of each simulation. The first four variables are intended as per-capita indicators (averaged values) and summarize the traveling experience of commuters, whereas the last one can be seen as a performance measure of the urban system.

\subsection{Experimental Results}

In this section, we show and discuss the results obtained from our simulations to compare them according to the just-mentioned indicators. We also provide some interesting insights concerning the impact of the three SMIs on people acceptance and on mobility resource usage levels.

\subsubsection{Number of Tangrhub Subscribers}

A subscriber is a person who uses the mobility services provided by tangrhubs. This value may measure the success of an SMI in terms of people acceptance. As noticeable in Figure 5, the number of subscribers increases as the SMI has more mobility resources, where the horizontal line denotes the entire population.

\subsubsection{Commuters' Performance Measures}

The first variable involved in the comparison is the traveled distance of commuters shown in Figure 6. In the three SMI simulations, commuters are expected to travel shorter distances than the pre-SMI ones even if the

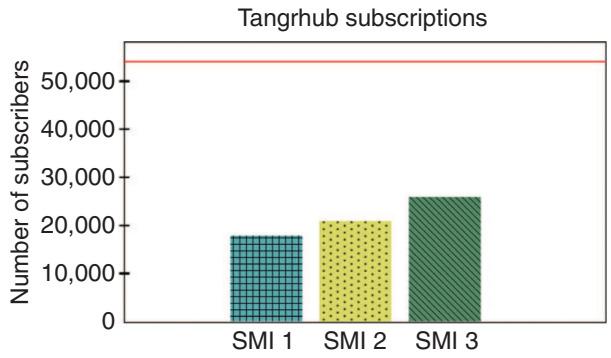

Figure 5: Tangrhub Subscriptions.

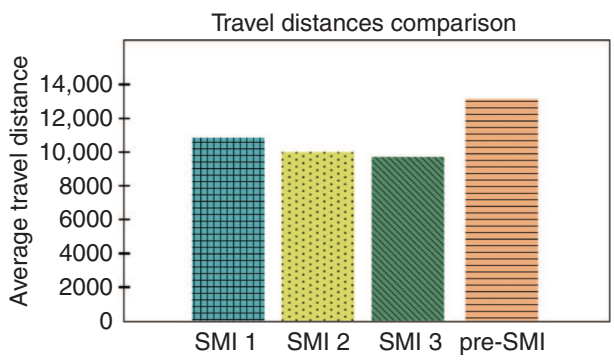

Figure 6: Traveled Distances. 
differences are not so marked. For what concerns travel times (Figure 7), in the simulations of SMI-1 and SMI-2, commuters spend less time traveling. This is a good indicator of the effectiveness of the SMIs. Conversely, in SMI-3, commuters spend much more time traveling than before. This indicates that SMI-3 has some problems either in the configuration or in moving people with respect to the pre-SMI.

Concerning the comparison of $\mathrm{CO}_{2}$ emissions produced by commuters, we found that the carbon footprint of the three SMI simulations tends to decrease in a directly proportional way to the number of subscribers (Figure 8). Therefore, we can affirm that the more the SMI satisfies a large section of the population, the more the simulation becomes eco-friendly if we use green vehicles. SMI-1, SMI-2, and SMI-3 reduce $\mathrm{CO}_{2}$ by $20 \%$, $25 \%$, and $35 \%$, respectively. Besides the environmental impact, we also found that there exists an inverse relationship between the number of subscriptions and the daily costs of mobility. As can be seen in Figure 9 , a commuter in the pre-SMI simulation spends on average 13.5 a day for traveling, whereas a commuter can satisfy his/her needs with a lower expense of 10, 9, and 8 in SMI-1, SMI-2, and SMI-3, respectively.

\subsubsection{Mobility Resource Usage}

Tangramob can provide statistics concerning the level of mobility resource usage of the SMIs (Figure 10). The analysis of these data allows understanding if an SMI is efficiently configured, so as to refine it for obtaining similar results with fewer mobility resources. In our case, it turns out that SMI-1 and SMI-2 are properly configured and their resources are used. SMI-3 has a large number of unused vehicles, so we can reduce its fleets

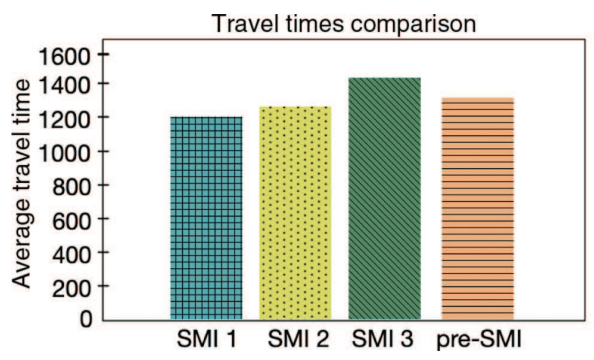

Figure 7: Travel Times.

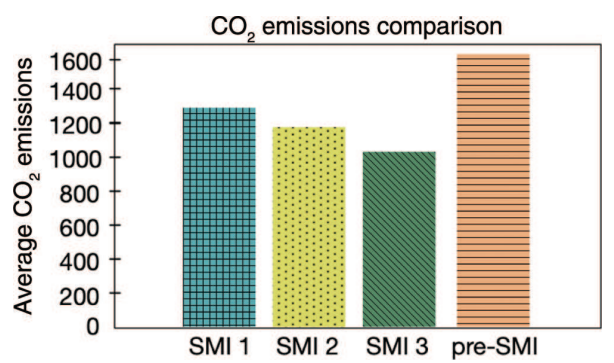

Figure 8: Emissions.

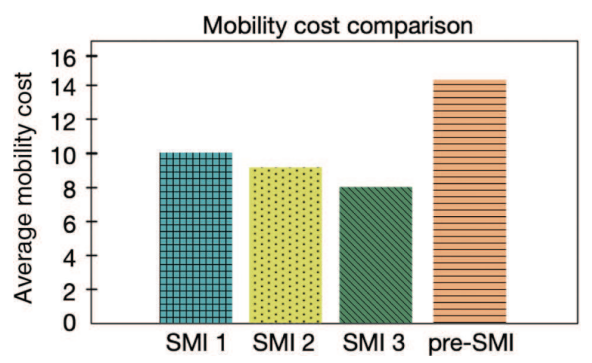

Figure 9: Mobility Costs. 


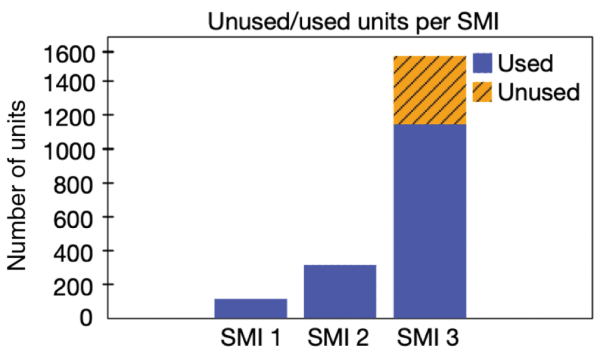

Figure 10: Mobility Resource Usage.

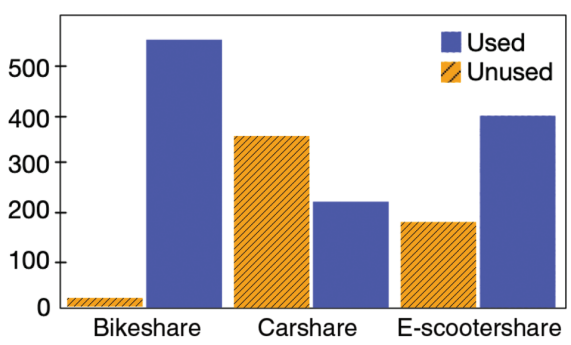

Figure 11: Mobility Resource Usage SMI-3.

in other simulation attempts. A closer look at the resource usage of SMI-3 in Figure 11 shows the incorrect sizing for the car and scooter services, highlighting, however, the right usage of bikes.

Gathering together all the results, we can conclude that a properly configured SMI helps in reducing several urban problems like traffic congestion levels and consequently air pollution. The experiment concludes that SMI-2 shortly reduced distances by $20 \%$, maintaining substantially the same travel times but significantly lowering the emissions and costs. Moreover, its application actually use all the resources associated with the services. The same conclusion can also be made for SMI-1 if the benefit is less noticeable. SMI-1 and SMI-2 could be evaluated in relation to their implementation costs by an urban planner and a decision maker. SMI-3, on the contrary, increases the travel time while maintaining important benefits in travel distances, emission, and costs. However, its implementation requires many resources, many of which are left unused.

The three simulations took about $2.5 \mathrm{~h}$ each, with 110 iterations on a Linux machine with an i7-7700 K CPU @ 4.8 GHz, a 5400 rpm hard drive, and 16 GB of RAM. We used in the test the whole 56,000 agent population. Running a mid-sized scenario with a real population of 500,000 inhabitants should be done, scaling the population to $10 \%$ as suggested in Ref. [11]; thus, such data make us confident about the feasibility of Tangramob simulations also for larger scenarios.

\section{Conclusions and Future Work}

Understanding how urban mobility is expected to evolve after the introduction of new smart mobility services is a crucial task in the urban planning field.

To address this problem, we introduced Tangramob, an agent-based simulation framework that allows users to assess the impacts and performances of a mobility initiative within an urban area of interest. Tangramob performs comparative experiments between before and after the introduction of a mobility initiative, approximating real-world urban dynamics by adopting reinforcement learning techniques. The computational nature of these experiments makes it easy to support urban mobility decisions, permitting to reduce costs and risks.

Although Tangramob is still under active development and improvement, the current version already permits running meaningful experiments that provide positive results on the usefulness and potentialities of the simulator. In particular, users can measure the impacts of a simulated SMI with respect to urban traffic levels, 
$\mathrm{CO}_{2}$ emissions, traveled distances, travel times, land use levels, cost of mobility, number of adopters, and resource usage level. Thus, it is up to the user to evaluate which variables are more relevant for understanding whether or not an initiative is in line with his/her objectives. The experiment we showed can help urban planners consider future initiatives and policies. SMI-1 is the cost-effective solution significantly impacting $\mathrm{CO}_{2}$ emissions and personal costs. SMI-2 is the most powerful initiative able to further lower those values while offering a variety of services to the commuters. SMI-3 is clearly oversized, and the improvements made possible by its use are not justified by the implementation costs and unused resource rate. From these, planners could refine SMI-1 and SMI-2 to arrive at a city planning simulation useful to decision makers.

Planned future work includes the extension of the current scoring function with additional traveling comfort criteria to measure the comfort of a traveling experience with a certain vehicle to let the commuter agents evaluate a mobility service as a whole. In addition, considering the lack of an already validated simulator sharing the same intents and modeling criteria of Tangramob, we are in the process of designing a pilot study on a medium-sized city in order to align the simulator to more realistic results.

\section{Bibliography}

[1] M. Adnan, F. C. Pereira, C. M. L. Azevedo, K. Basak, M. Lovric, S. Raveau, Y. Zhu, J. Ferreira, C. Zegras and M. Ben-Akiva, SimMobility: a multi-scale integrated agent-based simulation platform, in: 95th Annual Meeting of the Transportation Research Board Forthcoming in Transportation Research Record, The National Academies of Sciences, Engineering, and Medicine, Washington, DC, 2016.

[2] M. Behrisch, L. Bieker, J. Erdmann and D. Krajzewicz, SUMO - simulation of urban mobility: an overview, in: Proceedings of SIMUL 2011, The Third International Conference on Advances in System Simulation, IARIA, Barcelona, Spain, 2011.

[3] Catapult Transport Systems, Exploring the opportunity for mobility as a service in the UK, Transport Systems Catapult, Report, Catapult Transport Systems, UK, 2016.

[4] N. Cetin, A. Burri and K. Nagel, A large-scale agent-based traffic microsimulation based on queue model, in: Proceedings of Swiss Transport Research Conference (STRC), Monte Verita, CH, pp. 3-4272, Swiss Transport Research Conference, Switzerland, 2003.

[5] M. Ciuffini, C. Aneris, V. Gentili, S. Operto, L. Refrigeri and L. Trepiedi, La sharing mobility in Italia: numeri, fatti e potenzialitá, Osservatorio Nazionale Sharing Mobility (Italia), Report, Osservatorio Nazionale Sharing Mobility, Italy, 2016.

[6] M. Fellendorf and P. Vortisch, Microscopic traffic flow simulator VISSIM, pp. 63-93, Springer, New York, 2010.

[7] M. Finger, N. Bert and D. Kupfer, eds., From the Helsinki experiment to a European model? FSR Transport, Robert Schuman Centre for Advanced Studies (RSCAS) Research Project Reports, Firenze, Italy, 2015.

[8] I. García-Magarino, ABSTUR: an agent-based simulator for tourist urban routes, Expert Syst. Appl. 42 (2015), 5287-5302.

[9] I. García-Magarino, G. Palacios-Navarro, R. Lacuesta and J. Lloret, ABSCEV: an agent-based simulation framework about smart transportation for reducing waiting times in charging electric vehicles, Comput. Netw. 138 (2018), 119-135.

[10] B. Guo, Z. Wang, Z. Yu, Y. Wang, N. Y. Yen, R. Huang and X. Zhou, Mobile crowd sensing and computing: the review of an emerging human-powered sensing paradigm, ACM Comput. Surv. 48 (2015), 7.

[11] A. Horni, K. Nagel and K. W. Axhausen, The multi-agent transport simulation MATSim, Ubiquity-Press, London, 2016.

[12] M. R. Jabbarpour, R. M. Noor and R. H. Khokhar, Green vehicle traffic routing system using ant-based algorithm, J. Netw. Comput. Appl. 58 (2015), 294-308.

[13] H. Ma, D. Zhao and P. Yuan, Opportunities in mobile crowd sensing, IEEE Commun. Mag. 52 (2014), 29-35.

[14] E. Negri, “Salerno, un fallimento il servizio di bici condivise”, La cittá, Edizioni Salernitane, Italy, 2016.

[15] C. Pitas and D. Goodman, BMW tries to succeed where Daimler failed with London car-share scheme, Thomson Reuters, United States, 2014.

[16] K. Ramamohanarao, H. Xie, L. Kulik, S. Karunasekera, E. Tanin, R. Zhang and E. B. Khunayn, SMARTS: Scalable microscopic adaptive road traffic simulator, ACM Trans. Intell. Syst. Technol. 8 (2017), 26.

[17] United Nations, The world's cities in 2016, United Nations Human Settlements Programme, New York, 2016.

[18] R. A. Waraich, D. Charypar, M. Balmer and K. W. Axhausen, Performance improvements for large-scale traffic simulation in MATSim, pp. 211-233, Springer International Publishing, Cham, 2015. 